\title{
Dynamics of bacterial flow: Emergence of spatiotemporal coherent structures
}

\author{
Nicholas Sambelashvili, ${ }^{1}$ A.W.C. Lau, ${ }^{2}$ and David $\mathrm{Cai}^{3}$ \\ ${ }^{1}$ Department of Physics, New York University, New York, NY 10012, USA \\ ${ }^{2}$ Department of Physics, Florida Atlantic University, Boca Raton, FL 33431, USA \\ ${ }^{3}$ Courant Institute of Mathematical Sciences, New York University, New York, NY 10012, USA
}

(Dated: November 5, 2018)

\begin{abstract}
We propose a simple model of self-propelled particles to show that coherent structures, such as jets and swirls, can arise from a plausible microscopic mechanisms: (i) the elongated shape of the selfpropelled particles with (ii) the hardcore interactions among them. We demonstrate via computer simulation that these coherent structures, which emerge at sufficiently high densities of particles, have characteristics that are similar to those observed in recent experiments in bacteria baths.

PACS numbers: 87.18.Bb, 05.65.+b, 47.54.+r
\end{abstract}

Living systems often exhibit complex spatiotemporal patterns that are characteristic of many systems driven out-of-equilibrium [1]. Examples range from birds flocking 2] to internal organizations inside a cell [3]. A population of live microorganisms, such as bacteria like E. coli, suspended in an aqueous environment can provide a particular interesting model system to study pattern formation and collective motion in biology since the dynamics of the individual bacteria can be directly observed and critical parameters such as density and activity may be brought under experimental control [4]. These microorganisms continuously consume nutrients and dissipate the energy through the process of propelling themselves against the frictional force exerted on them by the fluid [5]. With a typical size of a bacterium of the order of microns and a typical speed of the order of $10 \mu \mathrm{m} / \mathrm{s}$, the Reynolds number $R \ll 1$ is quite small. Yet, a large concentration of these microorganisms constitutes a state that is far from equilibrium, and exhibits self-organized collective motion with spatial and temporal patterns that are both physically fascinating and potentially of great biological significance [6, 7, 8, 9]. As a first step towards understanding of how suspended cells generate coherent motion, we identify, in this Letter, two simple but central ingredients - the elongated shape of the self-propelled particles and the hardcore interactions among them, and demonstrate via computer implementation of these two ingredients that this system exhibits coherent jets and swirls with characteristics strikingly similar to those observed in experiments.

Recent experiments [6, 7, 8, 9] show that when concentrated, the crowd of swimming bacteria creates arrays of transient jets and swirls whose size can be orders of magnitude larger than an individual bacterium. These complicated, spatially coherent structures have been observed in Bacillus subtilis colony grown on an agar plate [6], in E. coli confined in a quasi-two-dimensional soap film 7], in Bacillus subtilis at the edge of a pendent drop 9]. It is estimated from direct visualization that these structures have a typical size of about ten times that of a bacterium and persist for a few seconds. More quantitative information about these structures may be extracted from a one-point passive microrheological tech- nique which tracks passive micron-sized beads dispersed in a bacterial bath of E. coli [7]. Interestingly, the meansquared displacement (MSD) of these passive beads exhibit superdiffusion at short time and diffusion at long time. It is measured that the crossover time $\tau_{c} \sim 2 \mathrm{~s}$ and the length $\ell_{c} \equiv \sqrt{\left\langle\left[\Delta x\left(\tau_{c}\right)\right]^{2}\right\rangle} \sim 10 \mu \mathrm{m}$ provide a natural time and length scales of these coherent structures, respectively.

There have been a few simulation models and theoretical treatments that aim to describe the above and related phenomena [2, 10, 11, 12]. Motivated by the patterns in fish schooling and bird flocking, Vicsek et al. numerically studied a model in which each particle (modelled as a point) moves at a constant speed and its direction is determined by averaging over the directions of a large collection of particles in its neighborhood plus a small random perturbation [2]. This model in 2D exhibits a nonequilibrium phase transition: At sufficiently high concentration, all particles spontaneously move in a single direction, thus breaking the rotational symmetry of the system. This phase transition is rationalized within a phenomenological dynamical xy-model [10]. This model further predicts that in 2D, the MSD of a tag particle exhibits superdiffusion at the transition. Indeed, via computer simulation of the bird flocking model, it was shown in Ref. [11] that a passive bead, interacting with a sea of "birds", exhibit superdiffusion as observed in Ref. 7]. However, the coherent structures appearing in the ordered state of the bird flocking model have a size scaled as the system size, whereas those observed in experiments have finite size. Note that the bird flocking model ignores the nematic-like ordering arising from the rod-like shape of a bacterium. More recently, a novel phenomenological macroscopic equations which take the ambient fluid and nematic ordering into account have been proposed to describe the dynamics of suspensions of self-propelled particles [12]. This theory is a generalization of the equilibrium hydrodynamics of nematogens to a non-equilibrium situation. It predicts that an ordered suspension of self-propelled nematics is unstable at long wavelength, possibly giving rise to vortices and jets. Although such macroscopic equations are well suited for probing structures at large scales, it seems rather difficult 

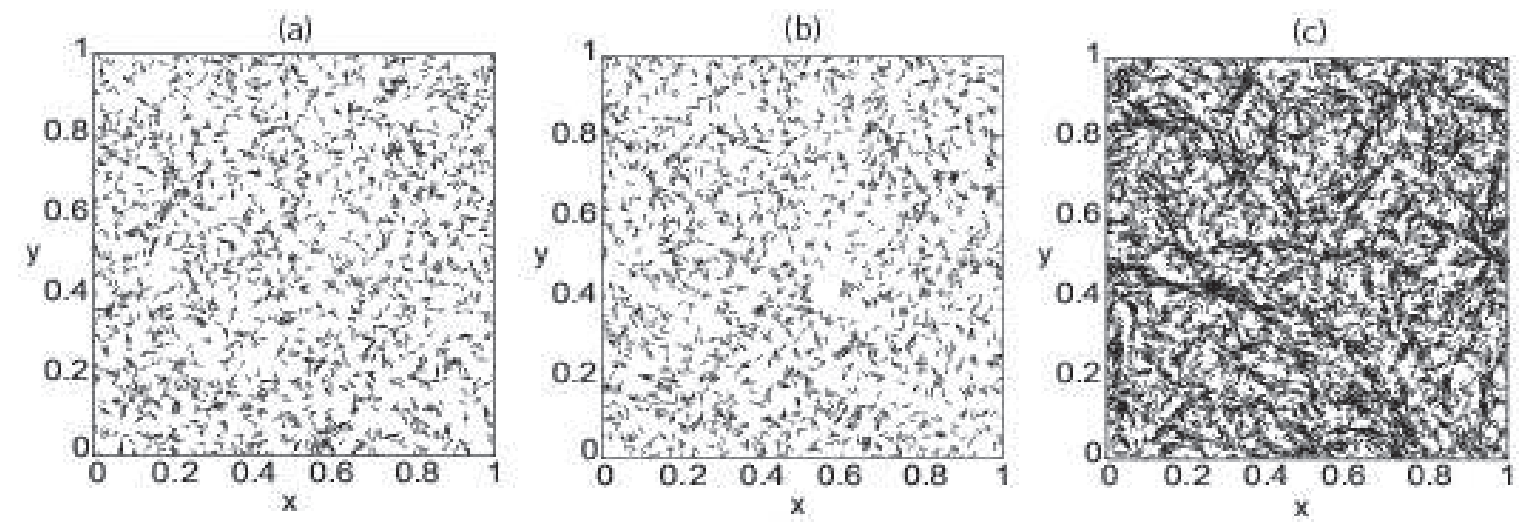

FIG. 1: Snapshots of bacterial motion at low and intermediate particle density. (a) and (b): The number of particles is $N=2000$. These two snapshots are one second apart. Each bacterium is represented as a vector whose length is the same as the bacterial length. There is no apparent coherent structure present. The bacteria move as if they were statistically independent, although some groupings occur accidentally, but they disappear quickly. The distribution is quite homogeneous and isotropic. (c) $N=8000$ - Emergence of coherent structures; note the dark stripes where a bunch of bacteria moves in similar directions.

to apply them to determine microscopic mechanisms for the coherent structures down to scales of a few particle sizes.

In this letter, we propose a new 2-D microscopic model that is based on two simple physical ingredients: First, since the ratio of the length of a bacterium to its width is typically $\sim 5$-10, we model each bacterium as an infinitely thin rod with a fixed length. Secondly, we impose hard-core interaction among them, i.e., they cannot intersect when they are moving. Thus, our model may be viewed as a nonequilibrium generalization of the twodimensional hard-core nematics studied in Ref. 13]. As we demonstrate below, our model in 2D exhibits coherent structures which emerge at sufficiently high densities, even though the bacteria in our model have no "will", in the sense that they do not seek out at what directions their neighbors are swimming, they do not communicate with each other via chemical signaling, nor do they interact hydrodynamically. Rather, the simple physical picture emerging from this study is that, with the constraints of the elongated shape of the needle and the excluded volume interaction, a self-propelled bacterium can find a new location only where it can fit into. This forces the needles to align locally and swim together in the form of swirls and jets. Our simulation shows that (i) typical sizes of these coherent structures are consistent with experimental observations, and (ii) the density fluctuations are characterized by non-Poisson statistics.

We now detail our model of bacterial bath and describe briefly our simulation method. We first deposit $N$ needles of length $\ell$, whose center-of-mass coordinates $\mathbf{r}_{i}=\left(x_{i}, y_{i}\right), i=1, \ldots, N$, are drawn at random from a uniform distribution in a $2 \mathrm{D}$ box with the length $L$. The orientation of each needle $\hat{\mathbf{n}}_{i}$ can be characterized by the angle with respect to the $y$-axis. This angle is chosen at random in the range of $[0,2 \pi]$ from a uniform distribution. At this stage, there may be many bacteria intersecting with each other. To impose the hard-core interactions, we must first obtain a non-intersecting state. This is accomplished by moving each needles by a certain distance and rotating its direction until no needles are intersecting. This state constitutes an acceptable initial state. We use the criterion in Ref. [13] to test whether two needles are intersecting: Two needles $i$ and $j$ intersect if and only if both $g_{i}$ and $g_{j}$ are negative, where the quantities $g_{i}$ and $g_{j}$ are defined as

$$
\begin{aligned}
& g_{i} \equiv\left(\mathbf{r}_{i j} \cdot \mathbf{v}_{i}\right)^{2}-(\ell / 2)^{2}\left[1-\left(\mathbf{v}_{i} \cdot \mathbf{v}_{j}\right)^{2}\right] \\
& g_{j} \equiv\left(\mathbf{r}_{i j} \cdot \mathbf{v}_{j}\right)^{2}-(\ell / 2)^{2}\left[1-\left(\mathbf{v}_{i} \cdot \mathbf{v}_{j}\right)^{2}\right],
\end{aligned}
$$

with $\mathbf{r}_{i j}=\mathbf{r}_{i}-\mathbf{r}_{j}$ and $\mathbf{v}_{i}$ and $\mathbf{v}_{j}$ being unit vectors perpendicular to $\hat{\mathbf{n}}_{i}$ and $\hat{\mathbf{n}}_{j}$, respectively. We employ periodic boundary condition in both directions. The bacterial motion is simulated with the following dynamics: a particle is chosen to move to a new position while remaining particles are fixed. Note that this asynchronous updating avoids complication associated with many-body collisions, which would be difficult to resolve computationally for the hardcore interactions. Thus, at each time step, an individual bacterium moves by a fixed distance $d$ and simultaneously its direction changes by a small angle $\theta$, which is chosen uniformly at random from the interval $[-\eta / 2, \eta / 2]$. If the chosen bacterium intersects with another one, in order to satisfy the hard-core constraint, we immediately discard the move and start a new trial move: we pull it back to the previous (original) position, and change its direction by another random angle $\theta$. If the number of such trials exceeds a preset number $N_{\text {trial }} \sim 40$ [14], then we increase $\eta$ by a small amount to a new $\eta^{\prime}=a \eta$, (say, $a=1.02$ ) and repeat the trial until this particular particle can make its move. Then we reset $\eta$ to its default value and update the next particle. We emphasize that there is no preferential searching involved in a sense that the bacterium is merely trying to find a 

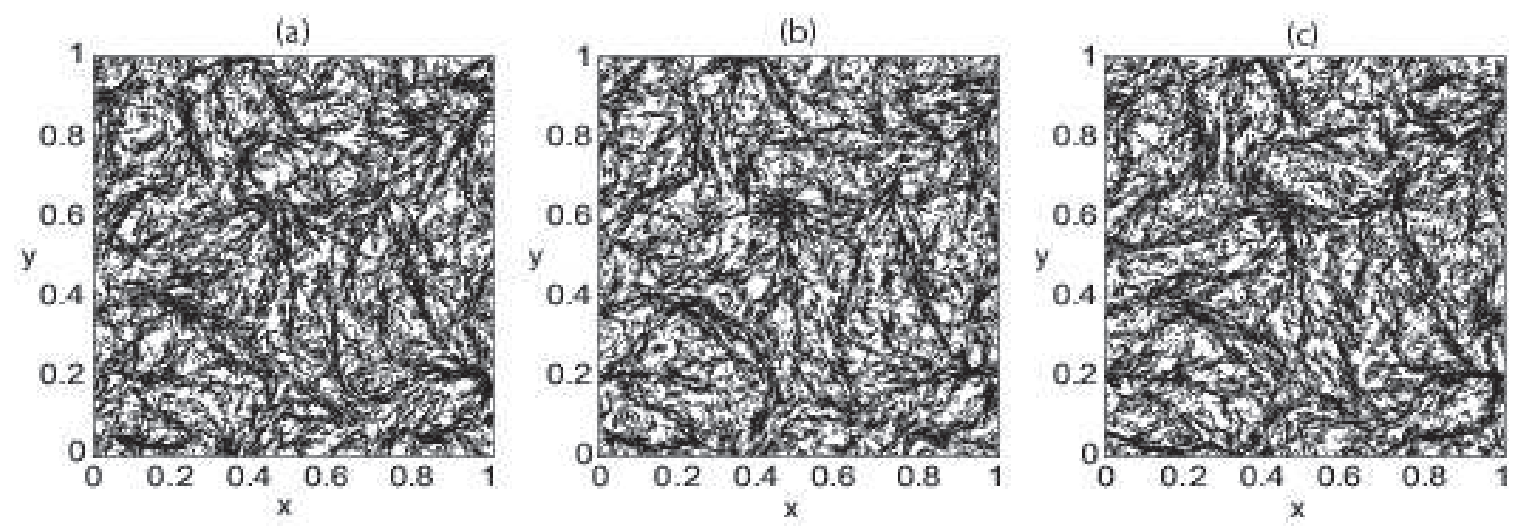

FIG. 2: Snapshots of bacterial motion at the high particle density $(N=12000)$. See Fig. (1) for the graphic representation convention. Dense needle regions form coherent structures of jets and swirls. Some of these structures can be seen to persist for seconds as shown here. (The time between each snapshot here is $1 \mathrm{~s}$.) Note the temporal evolution of the shape and size of these structures.

(a)

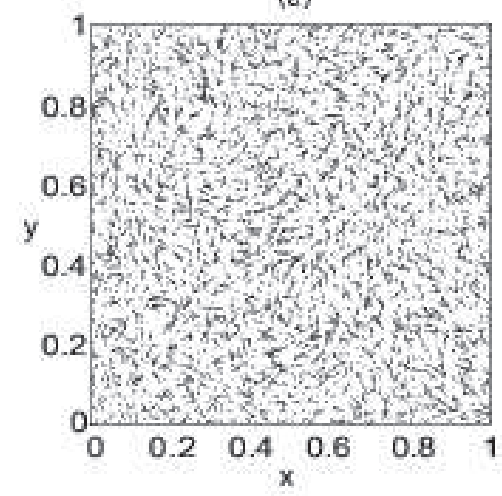

(b)

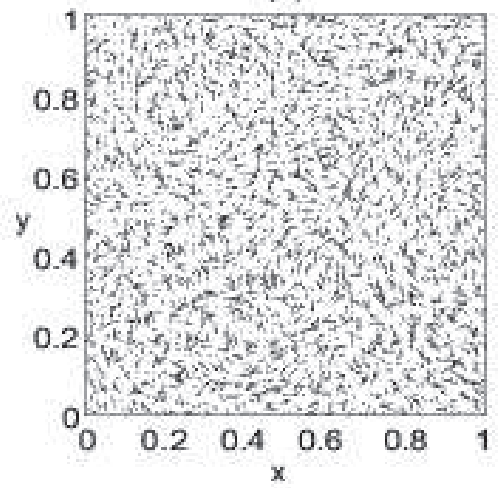

(c)

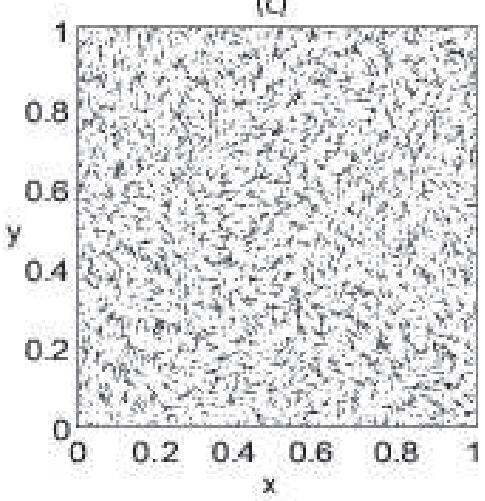

FIG. 3: Snapshots of velocity fields, $N=12000$, (see text). Long jets and large swirls are persistent. The size of jets is comparable to the box size; swirls can have long life-time (around $8-10$ seconds). The time between each snapshot here is 1 second with a total duration of two seconds shown.)

new location where it can fit into. This procedure to update the position and direction of the needle is repeated for all particles sequentially at each time step. We carry out our simulations, and discard the first $\sim 3000$ moves as transient. For all the simulations reported below, we have chosen $\ell=0.04, L=1, d=0.001$, and $N$ ranges from $\sim 10^{3}$ to $\sim 10^{4}$. The parameter $\eta$ determines how widely a bacterium can change its direction, and its default value is $\eta=0.01$. In order to compare with physical values, each time step corresponds to $\sim 0.01 \mathrm{~s}$. Roughly, this equivalently corresponds to the situation where a bacterium of length of a few microns swims at a typical speed of a few $\mu \mathrm{m} / \mathrm{s}[9]$.

We now turn to our main simulation results. Depicted in Figs. 1 and 2 are snapshots of the needle configuration for $N=2000,8000$, and 12000, respectively. As evident in these figures, different densities of particles result in different structures in their collective motion. For low densities, around $N \sim 10^{3}$ there are no spatiotemporally coherent structures, as shown in Fig. 1(a) and (b). Bacteria rarely collide. However, as the concentration of the particles reaches the values of $N \sim 4000$, some coherent structures start to emerge with a short persistent time $(t \ll 1 \mathrm{~s})$. At $N=8000$, these coherent structures become more prominent and one can easily identify long jets and small swirls (Fig. 1(c)). These structures can persist for a few seconds. For similar densities, the same structures are observed experimentally [7, 9]. As the particle concentration increases, the typical size of the coherent structures and the frequency of their occurrence also increase. The highest density we consider is $N=12000$. In this case, jets of the size of the box appear and almost all particles are involved in collective motion [Fig. 2. The evolution of the velocity field is shown in Fig. 3. where the velocity field is the average vector by summing bacterial vectors locally with the averaging area being $\ell^{2}$. Figure 3 clearly demonstrates the persistence of coherent structures for seconds in the velocity field. At a fixed particle concentration, the size of swirls clearly depends on $\eta$. As $\eta$ increases, the typical size of swirls decreases. This is because particles in the swirls at each time step explore wider angles and are able to escape 


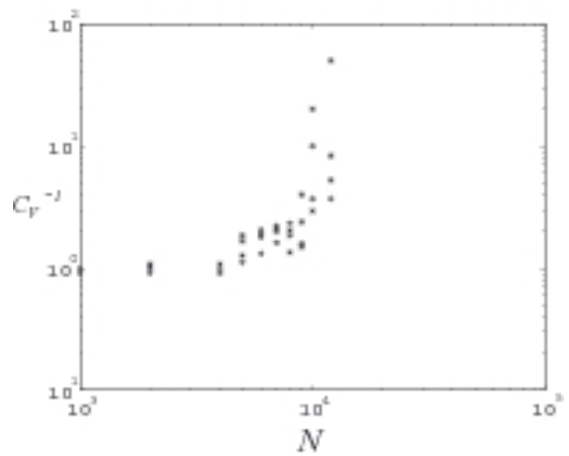

FIG. 4: Particle density fluctuation characterized by $C_{V}^{-1}$. $T_{C_{V}}=0.1 \mathrm{~s}$. For a fixed $N$, we plot here the value of $C_{V}^{-1}$ for each independent run. For example, at $N=8000$, there were three runs, thus three different values of $C_{V}^{-1}$. (Instead of averaging over these runs, we display this way to indicate the statistical spread.)

from the region of the swirl. For $\eta \sim 0.01$, the size of swirls is about $5-7$ times the lengths of the bacterium at $N \sim 4000-8000$. One of the temporal characteristics of jets or swirls is their persistence time. Experimental observations [9] indicate that the persistence time for swirls is on the order of several seconds, while jets can persist for longer time. Our simulations yield similar results for swirls and jets. Incidentally, for initial conditions with all parallel needles, our simulations show that this initial long-ranged order is quickly destroyed but the coherent structures of jets and swirls again develop as the system evolves. We emphasize that the hard-core interaction among elongated self-propelled objects underlies all these structures in the simulation. The physical picture emerges from this model is that the excluded-volume interactions effectively reduces $\eta$, which forces particles to align locally. This mechanism appears to be consistent with experimental observations in that bacteria tumble (hence, changing directions) less when concentrated 7].

To further quantify the transition from the homogeneous, isotropic flow to highly organized coherent motions of bacteria, we measure the coefficient of variance, $C_{V}$, from our simulation to describe the coherence of the motion, where $C_{V}=\sigma_{n}^{2} /\langle n\rangle,\langle n\rangle$ is the average number of bacteria appearing in a small square with an area $1 / 16$ over a fixed time interval $T_{C_{V}}$, and $\sigma_{n}$ is the number fluctuation over $T_{C_{V}}$. Figure 4 displays that $C_{V}^{-1}$ is nearly one at the low density limit (Note that $C_{V}=1$ for a Poisson statistics.). Then, $C_{V}^{-1}$ has a rapid rise around $N \sim 10^{4}$, signifying a drastic reduction of fluctuations in the particle density. Hence, the onset of the coherent motion of the bacterial flow.

In conclusion, we have proposed a possible mechanism for the emergence of coherent structures in a bacterial flow: they arise in $2 D$ from their elongated shapes with the hard-core interaction. We have shown that the model indeed gives rise to persistence of jets and swirls at sufficiently high density of needles, with comparable spatiotemporal scales as observed in experiments for bacteria at similar densities. When the bacterial concentration becomes sufficiently high, one may suspect that hydrodynamic interactions of solution become important. As noted before, our simple model does not taken into account hydrodynamic interactions. In general, inclusion of hydrodynamic interactions for elongated objects is computationally involved. However, we have performed a simulation of spherical particle motion following method in Ref. 15 inside a $3 D$ unit box, taking into account of the lubrication effect only. In this setup, we did not observe any jets or swirls, which means that hydrodynamics alone probably cannot organize spherical particles into large-scale coherent motions. This is indicative of the fact that the elongated shape together with hardcore interaction in $2 \mathrm{D}$ plays a primary role for the emergence of coherent structures even in the presence of hydrodynamics. We believe that at high densities the formation of the coherent structures due to our mechanism is robust qualitatively, with possible quantitative modifications by details of hydrodynamic interactions.

We acknowledge fruitful discussion with Daniel T. Chen, T.C. Lubensky. This work was supported by the NSF through the MRSEC Grant DMR-0079909 for A.W.C.L., DMS-0206679 and DMS-0507901 for N.S and D.C.
[1] J.P. Gollub and J.S. Langer, Rev. Mod. Phys. 71, S396 (1999).

[2] T. Vicsek et al., Phys. Rev. Lett. 75, 1226 (1995).

[3] F.J. Nedelec et al., Nature 389, 305 (1997).

[4] H.C. Berg, Random Walk in Biology, (Princeton, NJ, 1993); Physics Today 53, 24 (2000).

[5] E.M. Purcell, Am. J. Phys. 45, 3 (1977).

[6] Neil H. Mendelson et al., J. of Bact. 181, 600 (1999).

[7] X.L. Wu and A. Libchaber, Phys. Rev. Lett. 84, 3017 (2000).

[8] G.V. Soni at al, Biophys. J. 84, 2634 (2003).

[9] C. Dombrowski et al., Phys. Rev. Lett. 93, 098103
(2004).

[10] J. Toner and Y. Tu, Phys. Rev. Lett. 75, 4326 (1995); Phys. Rev. E 58, 4828 (1998).

[11] Cuillanume Gregoire et al., Phys. Rev. E 64, 011902 (2001).

[12] R. Aditi Simha and Sriram Ramaswamy, Physica A 306, 262 (2002); Phys. Rev. Lett. 89, 058101 (2002).

[13] D. Frenkel and R. Eppenga, Phys. Rev. A 31, 1776 (1985).

[14] Note that we have chosen $N_{\text {trials }} \sim 40$ in order to have as few particles as possible to be stuck in some region. Typically, the fraction of stuck particles does not exceed 
$10 \%$.

[15] R.C. Ball and J.R. Melrose, Physica A 247, 444 (1997). 